\title{
ANALYSIS OF THE QUALITY OF MOISTENING OF THE SINTER MIX IN THE DRUM PELLETIZER
}

\author{
Sergey Krivenko ${ }^{l)^{*}}$ \\ 1) Mariupol State University, Faculty of Law and Economics, Mariupol, Ukraine
}

Received: 15.10 .2018

Accepted: 21.01.2019

* Corresponding author:e-mail: booktwix@gmail.com Tel.: +380 96757 4547, Department of Mathematical Methods and System Analysis, Faculty of Law and Economics, 129a, Budivelnykiv Ave., Mariupol, Ukraine, 87500

\begin{abstract}
The analysis of changes in moisture, equivalent diameter of the granules, layer porosity and size variation under the abrupt perturbing effect on the input humidity of the charge was made. The change in other parameters from the charge moisture lags about 30 seconds. This is due to different mass transfer rates for moisture and fine fractions. PI-controller settings for control of all measured parameters were calculated. The best indicators of the transition process comply with the control of moisture content of the charge. However, the most representative is the simultaneous control of these parameters. A new indicator of the effectiveness of the shower unit of the pelletizer drum is developed, based on the difference in the rates of mass transfer of moisture and solid particles. The value of this indicator should be kept to a minimum.
\end{abstract}

Keywords: pelletizer, moisture, equivalent diameter, porosity, variation

\section{Introduction}

The most efficient modes of sintering, which correspond to Voice principle (equality of speeds of movement of the fronts of heat transfer and combustion of fuel mixture) to the success of the process of agglomeration of iron ore materials is necessary to ensure [1,2]. Production of highquality sinter and high performance is impossible without proper control of the gas-dynamic properties of the sintered layer. Pelletizing, which forms the final fractional composition of the charge before loading onto the pallets of the sintering machine, is one of the main technological stages of preparation of the charge for sintering. The quality of pelletizing depends on many factors: the fractional composition of the initial charge and its hydrophilic properties, the amount of moisture in the pelletizer, the properties of the shower device and the pelletizer drum, etc. Extensive method by increasing the drum length up to $12 \mathrm{~m}$. to improve the fractional characteristics of the charge is used most often. Of course this has a positive effect, but does not exclude the use of intensive methods. Therefore, works aimed at improving the quality of pelletizing are relevant and require further development.

The purpose of the article is to study the methods of pelletizing control in the pelletizer drum and to assess of the moisturing quality in it.

\section{Experimental programme}

A study of the influence of humidity on the final particle size distribution of the charge and on the parameters of the layer was carried out at PJSC "MK "AZOVSTAL" [3]. Length of the drum 
pelletizer $-6 m$, diameter $-2.5 \mathrm{~m}$, the angle of the axis $-0^{\circ}$, productivity -220 tons / hour. The current water consumption for moisture was $6.5 \mathrm{~m}^{3} /$ hour. The moisture content $W$ of the charge was increased from 4.48 to $8.3-9.1 \%$, the equivalent diameter $d_{e}$ - from 1.04 to $3.5-4.2 \mathrm{~mm}$. The variation of size $V$ is decreased from 2.75 to $0.52-0.62 \mathrm{~mm} / \mathrm{mm}$.

The transit time of materials through the pelletizer and intermediate bunker was $75 \mathrm{sec}$. A heating of the charge by the heat of hot returned agglomerate allowed raising the temperature of the pelletized charge to $65^{\circ} \mathrm{C}$. In the course of studies, the water consumption for pelletizing of the charge was increased in comparison with the established service personnel by $0.5 \mathrm{~m}^{3} / \mathrm{hour}$ $(\Delta W=0.35 \%)$. The equivalent diameter $d_{e}$ and the coefficient of variation $V$ of the particle size of the granules of the pelletized sinter charge was determined by mid-logarithmic formulas $[4,5]$.The measured parameters depend on many probabilistic factors, so it is not possible to stabilize them at the same value at the initial time. The equivalent diameter values were determined for the samples by sieving. The porosity was determined using a grid mathematical model based on the known fractional composition.Curves of change of the layer porosity, humidity of pelletized charge, pellets equivalent diameter and coefficient of variation, are presents by Fig. 1. The curves are obtained by the area method for the models presented below.

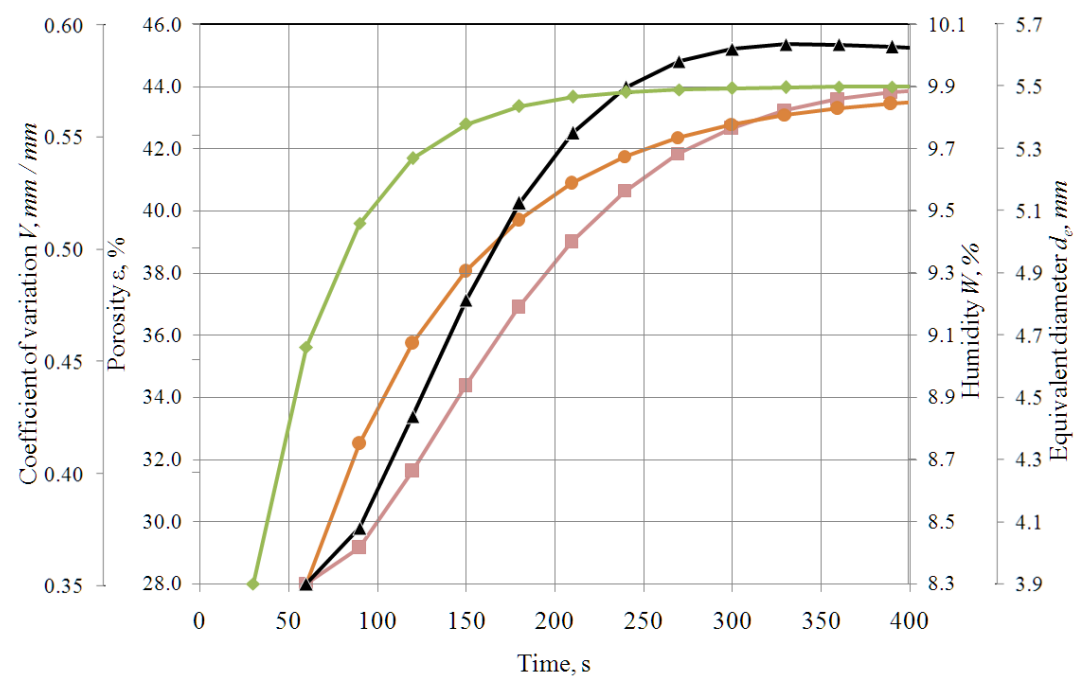

Fig. 1 Dynamics of changes in porosity $(\bullet)$, humidity $(\bullet)$, equivalent diameter of particles ( $(\mathbf{\bullet})$ and coefficient of variation $(\boldsymbol{\Delta})$ pelletized charge with the increase in water flow

It was found that when the changing humidity is from 8.3 to $9.9 \%$ the value of the layer porosity is varies growing from 28 to $45 \%$, the mid-logarithmic diameter of granules - from 3.9 to $5.5 \mathrm{~mm}$, the variation of size - from 0.35 to 0.57 . Porosity and mid-logarithmic diameter of the granules increased and variation decreased when you add the humidity. This indicates low humidity of the pelletized charge. Due to the fact that the transition processes were investigated, the pelletizing parameters did not have a steady value and the humidity value, which corresponds to the maximum porosity of the layer, cannot be claimed.

The value of the coefficient of linear correlation between the layer porosity and the coefficient of size variation was $R=-0.62$. That is, the correlation is not high. This is due to the fact that the value of porosity depends on the deviation of small fractions relative to the dominant one, and the variation characterizes the deviation relative to the equivalent diameter. The values of the 
dominant fraction and the equivalent diameter of the granules for bulk materials do not coincide.The correlation coefficient is negative because the size variation and the porosity change in inverse relationship $\varepsilon=f(1 / V)$.

Based on measured data, it was found that with an increase in the water flow rate in pelletizer, the humidity of the charge at the outlet of the intermediate hopper of the loading device is stabilized within $\tau_{W}=150-200 \mathrm{~s}$ and increases by $0.35 \%$ abs. The delay time was $\approx 30 \mathrm{~s}$.

It is necessary $\tau_{\varepsilon}=250-300 \mathrm{~s}$ to stabilize the equivalent particle diameter, layer porosity and coefficient of variation. The equivalent diameter is increased by $\approx 1.5 \mathrm{~mm}$, porosity - by $5.5 \%$ abs, coefficient of variation - by 0.06 . The delay time is the same for all three of these parameters and is $\approx 60 \mathrm{~s}$. Time lag of stabilization of new values of porosity, equivalent diameter of granules and coefficient of variation from humidity $\Delta \tau=\tau_{\varepsilon}-\tau_{W}=100 \mathrm{~s}$, probably due to the following.

According to research Korotich V. I. a characteristic feature of the motion of the charge in the mode of rolling is the formation of "bud" around which the rotation of material occurs [6]. Experiments have shown that the material is segregated by size when moving: small fractions are concentrated near this point. Crumbling material moves in layers of thickness, which is equal to the average diameter of the granules. Thus, the first portions of water are transported to the pelletizer discharge end by large fractions faster than the mass transfer of small particles from the buds middle occurs. The phenomenon of the uneven distribution of water when the cost change is also confirmed by other researchers, who found that for pulsating water flow in pelletizer observed delamination of the granules.

\section{Discussion of results}

The aperiodic (inertial) first-order equation with lag to describe the transients of the drum pelletizer made [7]

$$
W(p)=\frac{K_{o b}}{T_{o b} p+1} \cdot e^{-p \tau_{l a g}} ; h(t)=K_{o b} \cdot\left(1-e^{-\frac{t}{T_{o b}}}\right) \cdot 1\left(t-\tau_{\text {lag }}\right) .
$$

where: $W(p)$ - transfer function

$p$ - Laplace operator

$K_{o b}-$ the gain

$T_{o b}\left[s^{-1}\right]$ - the time constant

$\tau_{\text {lag }}[s]$ - the lagging time

$h(t)$ - the equation of the curve of acceleration

$t[s]$ - the time

$1\left(t-\tau_{\text {lag }}\right)$ - single function with delay

Dynamic parameters of the pelletizer drum were determined by the area method (Table 1) [8]. Indices at coefficients in Table 1 correspond to the transfer functions (1) of the output moisture of the charge $W$, of the layer porosity $\varepsilon$, of the equivalent diameter of the granules $d_{e}$ and of the coefficient of variation $V$ from the water flow to the pelletizer.

Table 1 Characteristics of the drum-pelletizer

\begin{tabular}{|l|c|c|c|}
\hline & $\boldsymbol{K}_{\boldsymbol{o b}}$ & $\boldsymbol{T}_{\boldsymbol{o b}}, \boldsymbol{s}^{-\mathbf{1}}$ & $\boldsymbol{\tau}_{\text {lag }}, \boldsymbol{s}$ \\
\hline Humidity $W, \%$ & $K_{W}=42.17 \% /\left(\mathrm{m}^{3} / \mathrm{s}\right)$ & $T_{W}=46.50$ & $\tau_{W}=30$ \\
\hline Porosity $\varepsilon, \%$ & $K_{\varepsilon}=662.651 /\left(\mathrm{m}^{3} / \mathrm{s}\right)$ & $T_{\varepsilon}=88.20$ & $\tau_{\varepsilon}=60$ \\
\hline Equivalent diameter $d_{e}, \mathrm{~mm}$ & $K_{d}=180.72 \mathrm{~mm} /\left(\mathrm{m}^{3} / \mathrm{s}\right)$ & $T_{d}=121.95$ & $\tau_{d}=60$ \\
\hline Size variation $V, \mathrm{~mm} / \mathrm{mm}$ & $K_{V}=-7.231 /\left(\mathrm{m}^{3} / \mathrm{s}\right)$ & $T_{V}=89.4$ & $\tau_{V}=60$ \\
\hline
\end{tabular}


Gain factors are overestimated due to the fact that the water flow rate is specified in the SI system. For more production-specific units, their values must be divided by $3600 \mathrm{~s}$. In addition, given the extreme nature of the change in porosity and in the equivalent diameter from the moisture content of the pelletized charge, the transfer coefficients of the object will have different values, depending on the properties of the charge and other parameters. Therefore, the effect of moisture on the layer should be considered in a small range. Changes in the transmission coefficients depending on the properties of the charge and humidity must be taken into account when controlling.

Note that the values of the constant time for porosity and variation of size isequal, which indicates their high correlation.

Also, despite the growth of the equivalent diameter, the size variation and porosity of the layer stabilize faster, which is explained by the narrowing of the range of granules diameters. Based on the values of time constants, the size variation stabilizes slightly longer.

A more accurate description of transition processes to calculate the right values of indicator of the quality of hydration necessary. Use of other laws changes is possible [7]:

- $\quad$ aperiodic (inertial) link of the second order with delay

$W(p)=\frac{K_{o b}}{\left(T_{3} p+1\right)\left(T_{4} p+1\right)} \cdot e^{-p \tau_{l a g}}, h(t)=K_{o b} \cdot\left[1-\frac{1}{T_{3}-T_{4}}\left(T_{3} e^{-\frac{t}{T_{3}}}-T_{4} e^{-\frac{t}{T_{4}}}\right)\right] \cdot 1\left(t-\tau_{\text {lag }}\right)$

where: $T_{3}$ and $T_{4}\left[s^{-1}\right]-$ the time constant

- $\quad$ oscillating link with an additional component with delay

$$
W(p)=\frac{K_{o b}}{p\left(T^{2} p^{2}+2 T \xi p+1\right)} \cdot e^{-p \tau_{\text {lag }}}, h(t)=K_{o b} \cdot\left[1-C e^{-\gamma t} \sin (\lambda t+\theta)\right] \cdot 1\left(t-\tau_{\text {lag }}\right),
$$

where: $0 \leq \xi<1 ; C=\frac{1}{\lambda T} ; \theta=\operatorname{arctg} \frac{\lambda}{\gamma} ; \gamma=\frac{\xi}{T} ; \lambda=\frac{\sqrt{1-\xi^{2}}}{T}$.

The calculated values of the coefficients of the formula (3) are shown in Table 2.

Table 2 Values of pelletizer model (3) coefficients

\begin{tabular}{|c|c|c|c|c|}
\hline \multirow{2}{*}{} & \multicolumn{4}{|c|}{ The curve parameters of acceleration } \\
\cline { 2 - 5 } & $\boldsymbol{W}$ & $\boldsymbol{d} \boldsymbol{e}$ & $\boldsymbol{\varepsilon}$ & $\boldsymbol{V}$ \\
\hline $\mathrm{T}=$ & 60.24 & 69.22 & 57.03 & 57.47 \\
\hline$\xi=$ & 0.39 & 0.88 & 0.77 & 0.78 \\
\hline $\mathrm{C}=$ & 1.08 & 2.11 & 1.58 & 1.59 \\
\hline$\lambda=$ & 0.0153 & 0.0068 & 0.0111 & 0.0109 \\
\hline$\gamma=$ & 0.0064 & 0.0127 & 0.0136 & 0.0135 \\
\hline$\theta=$ & 1.17 & 0.49 & 0.69 & 0.68 \\
\hline
\end{tabular}

The settings of the PI controller corresponding to the degree of oscillation $\mathrm{m}=0.351$ for control using various controlled input parameters are determined by the method of extended frequency response (Table 3). 
Table 3 Results of the regulation of the controlled parameters

\begin{tabular}{|c|c|c|c|c|c|c|c|c|c|c|c|c|c|c|}
\hline \multirow[b]{3}{*}{$\begin{array}{l}\text { Para- } \\
\text { meter }\end{array}$} & \multicolumn{2}{|c|}{ Regulator** } & \multicolumn{12}{|c|}{ Control quality } \\
\hline & \multirow[b]{2}{*}{$K_{p} \cdot 10^{-3}$} & \multirow[b]{2}{*}{$\begin{array}{c}T_{i} \cdot 10^{-4} \\
s^{-1}\end{array}$} & \multicolumn{4}{|c|}{ Overshoot, \% } & \multicolumn{4}{|c|}{ Regulation time, $s$} & \multicolumn{4}{|c|}{ Maximum deviation } \\
\hline & & & $\boldsymbol{W}$ & $d_{e}$ & $\varepsilon$ & $\boldsymbol{V}$ & & $d_{e}$ & $\varepsilon$ & $\boldsymbol{V}$ & $\begin{array}{l}W, \\
\%\end{array}$ & $\begin{array}{c}d_{e} \\
\boldsymbol{m m}\end{array}$ & $\begin{array}{l}\varepsilon, \\
\%\end{array}$ & $\begin{array}{c}V, \\
\frac{m m}{m m}\end{array}$ \\
\hline$W$ & & & 20.0 & 16.0 & 3.6 & 18.7 & 670 & 85 & 680 & 680 & & & & -0.38 \\
\hline$d_{e}$ & 0.17 & & 22.0 & 20.0 & 21.7 & 21.5 & 1230 & 1450 & 1300 & 1300 & 0.0 & 1 & & -0.50 \\
\hline$\varepsilon$ & 1 & & 20.8 & 18.1 & 20.0 & 19.7 & 1400 & 1600 & 1450 & 1450 & 0.036 & 1.14 & 4. & -0.52 \\
\hline$V^{*}$ & 12.5 & 13.6 & 20.8 & 17.8 & \begin{tabular}{|l|}
19.3 \\
\end{tabular} & 20.0 & 1300 & 1600 & 1270 & 1270 & 0.036 & 1.13 & 4.7 & -0.51 \\
\hline
\end{tabular}

* - with positive feedback

**- $K_{p}, T_{i}$ - the proportional and integral setting

Quality indicators of regulation of the pelletizer drum for each controlled parameter are investigated (Table 3 and Fig. 2). The settings of the PI-controller corresponding to $20 \%$ overshoot of each of the parameters were consistently set and controlled changes in the remaining parameters. Based on this, we determined the quality of transients for them.

From the research results it follows that the overregulation changes insignificantly for all variants. However, the humidity control of the charge at the outlet of the pelletizer has the best characteristics. At the same time, the duration of the transition process and deviations of all parameters are the smallest. This control is the most optimal for stable raw charge materials. Nevertheless, the humidity at the fluctuating initial dosing and fractional composition of the charge should provide maximum gas permeability. In addition, the measurement error is affected by hydrated moisture. Therefore, the porosity of the layer, the equivalent diameter of the granules and the variation of their size to regulate and find the maximum gas permeability are more preferred. They are also more closely related to the productivity of the agglomeration process.

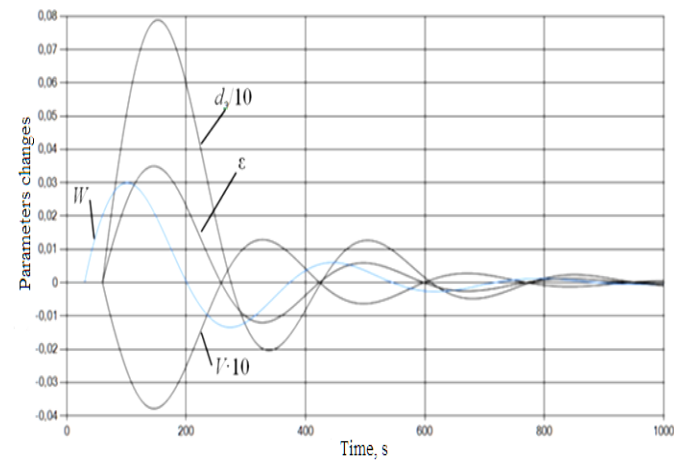

(a)

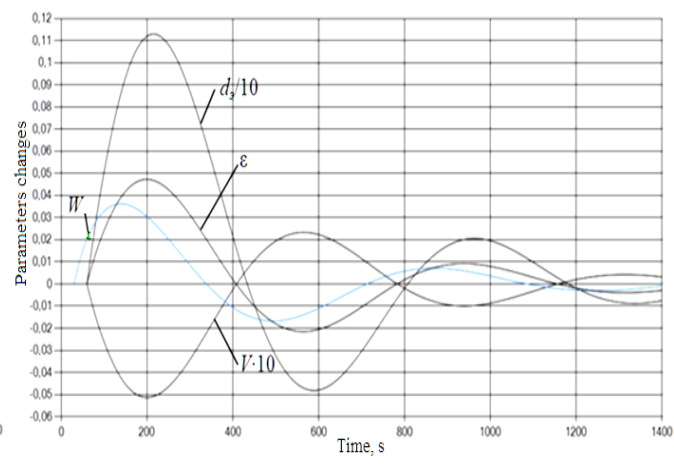

(b)

Fig. 2 Transients in the control of humidity of the pelletized charge (a) and porosity of the formed layer (b)

It is found that the regulation of the equivalent diameter of the granules has insignificantly better regulation. But given the fact that the maximum equivalent diameter corresponds to the formation of waterlogging, it is necessary to take into account all three parameters in the interaction [9-11]. 
The effective length of the pelletizer drum, on which the most intensive growth of granule size occurs, depends on the parameters of the shower unit inside. Typically, the moistening is carried out on $1 / 3$ of the length of the pelletizer from the entrance. Based on the fact that the moisture content of the charge at the outlet is changing much faster than the porosity of the layer, the variation in size and, especially, the equivalent diameter, it follows that the method of supplying moisture to the charge requires improvement.

The relative difference $\Delta S$ between the areas above the normalized curves of acceleration of humidity $S_{W}$ and equivalent diameter $S_{d}$ (or porosity $S_{\varepsilon}$ ) to assess the quality of moisturing can be used (Fig. 3)

$$
K_{y}=\frac{S_{d}-S_{W}}{S_{W}} .
$$

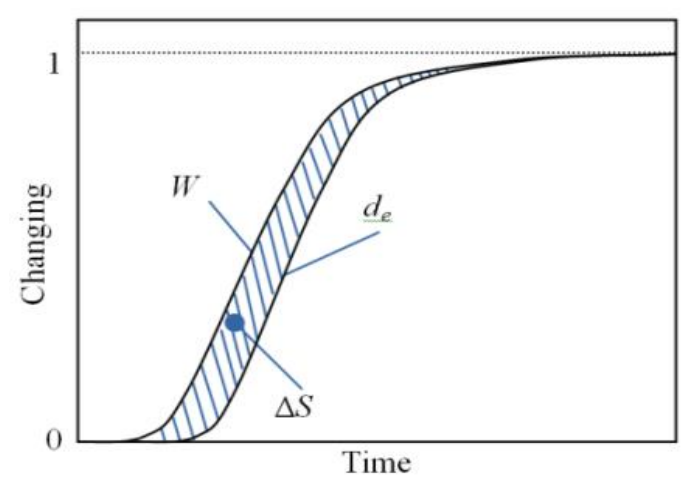

Fig. 3 Explanation of the formula (4)

The proposed coefficient shows the deviation of the acceleration curves by the parameters of the layer and the fractional composition of the charge relative to the base acceleration curve by humidity. Inefficient moisturizing corresponds to the irrational distribution of water on the surface of the material inside the pelletizer. More intensive mass transfer of lumpy fractions under the action of hygroscopic adhesion forces corresponds to the zero value of the index $K_{y}$.

For an aperiodic (inertial) link (1), the area above the acceleration curve $\mathrm{h}(\mathrm{t})$ is

$$
S=\int_{0}^{\infty}(1-h(t)) d t=\int_{0}^{\infty} e^{-\frac{t}{T_{o \sigma}}} d t=\left.T_{o b} e^{-\frac{t}{T_{o \sigma}}}\right|_{0} ^{\infty}=T_{o b} .
$$

Taking into account the lag time $S=T_{o b}+\tau_{\text {lag }}$. Accordingly, the indicator $K_{y}$ by the equivalent diameter is equal to

$$
K_{y}=\left(T_{d}+\tau_{d}-T_{W}-\tau_{W}\right) /\left(T_{W}+\tau_{W}\right) .
$$

Similarly, for the model of the drum (2)

$$
S=\int_{0}^{\infty} \frac{1}{T_{3}-T_{4}}\left(T_{3} e^{-\frac{t}{T_{3}}}-T_{4} e^{-\frac{t}{T_{4}}}\right) d t=T_{3}+T_{4}
$$

and given the time lag $S=T_{3}+T_{4}+\tau_{3 a n}$. Then 


$$
K_{y}=\frac{T_{3 d}+T_{4 d}+\tau_{d}-T_{3 W}-T_{4 W}-\tau_{W}}{T_{3 W}+T_{4 W}+\tau_{W}} .
$$

For the model (3)

$$
S=\int_{0}^{\infty} C e^{-\gamma t} \sin (\lambda t+\theta) d t=C \frac{\lambda \cdot \cos (\theta)+\gamma \cdot \sin (\theta)}{\gamma^{2}+\lambda^{2}} .
$$

$K_{y}$ is determined after substitution of the corresponding values of the areas calculated by the formula (9).

The value of the index $K_{y}$ at the same time step of measurements of humidity $W_{i}$ and equivalent diameter $d_{i}$ (porosity $\varepsilon_{i}$, variations of size $V_{i}$ ) can be determined approximately on the basis of the trapezoid method [12]

$$
S=n-\left(\frac{h_{0}+h_{n}}{2}+\sum_{i=1}^{n-1} h_{i}\right)
$$

where: $n$ - the number of the last dimension, starting from 0 .

\begin{tabular}{|c|c|c|c|c|}
\hline \multirow{2}{*}{\multicolumn{2}{|c|}{ Link type }} & \multicolumn{3}{|c|}{$K_{y}$} \\
\hline & & $d_{e}$ & $\varepsilon$ & $\boldsymbol{V}$ \\
\hline \multicolumn{2}{|c|}{ Aperiodic (inertial) link of the first order with lag } & 1.38 & 0.94 & 0.95 \\
\hline \multicolumn{2}{|c|}{ Oscillatory link with additional and-component with lag } & 1.38 & 0.94 & 0.95 \\
\hline \multirow{2}{*}{ The method of trapezes } & Value & 1.47 & 1.01 & 1.03 \\
\hline & Error $*, \%$ & 6.73 & 7.86 & 7.85 \\
\hline
\end{tabular}

The values $K_{y}$ calculated by different methods are presented in Table 4.

Table 4 Values of the index $K_{y}$

* - the true value of the $K_{y}$ corresponds to the oscillatory link with an additional I-component with a lag

It follows that the trapezoid method allows estimating the moisturing parameters with a satisfactory error.

\section{Conclusion}

The quality of the charge pelletizing in the drum depends to a significant extent on the correspondence of the water mass transfer rates and fine solid particles between the granules. The lagging to $30 \mathrm{~s}$ behind the rate of change of the equivalent diameter of the granules, the porosity of the layer and the variation of size from humidity is observed. The mismatch of these speeds reduces the effective length of the pelletizer drum. The coefficient characterizing the shower unit was developed. Its value for the industrial drum is $0.94-1.38$ depending on the compared parameters. The minimum value of which increases the effective length of the pelletizer by $3 \mathrm{~m}$.

\section{References}

[1] E. W. Voice, R. Wild: J. Metals, 1958, No. 2, p. 105-110 
[2] Sheng-li Wu, Juan Zhu, Ji-cheng Bei, Guo-liang Zhang, Xiao-bo Zhai: International Journal of Minerals, Metallurgy and Materials, Vol. 22, No 9, 2015, p. 907-915, DOI: $10.1007 / \mathrm{s} 12613-015-1149-0$

[3] S. V. Krivenko: Metallurgical processes and equipment: International scientific-technical and production journal, 2011, No 2(14), p. 22-26

[4] S. G. Bratchikov, Y. L. Berman, J. L. Belotcerkovsky: Thermotechnics of iron ore sintering, Moscow, 1970

[5] S. V. Krivenko: News of Higher educational institutions. Ferrous metallurgy: Scientific, technical and production journal, 2010, No. 8, p. 7-11

[6] V. I. Korotych, Yu. A. Frolov, G. N. Bezdezhsky: Agglomeration of ore materials, Yekaterinburg, 2003

[7] I. M. Makarov, I. M. Mensky: Linear automatic systems (theory elements, calculation methods and reference material), second ed., Moscow, 1982

[8] Kh. Guretskiy: Analysis and synthesis of control systems with delay, Moscow, 1974

[9] S. V. Krivenko: Metal and casting of Ukraine: Scientific journal, 2011, No. 8, p. 25-29

[10]P. Kapur: International Journal of Mineral Processing, 2003, No 72, p. 417-427, DOI: 10.1016/S0301-7516(03)00116-9

[11] Yu. A. Frolov: Stal, 2009, No. 8, p. 2-7. DOI:10.3103/S0967091209080099

[12] Atkinson, Kendall E.: An Introduction to Numerical Analysis, second ed., New York, 1989

\section{Acknowledgements}

The article is made in cooperation with the Department of iron metallurgy of Pryazovskyi State Technical University (Mariupol, Ukraine). 